\title{
Erratum to: Incidence, risk, management, and outcomes of iatrogenic full-thickness large bowel injury associated with 56,882 colonoscopies in 14 Lithuanian hospitals
}

\author{
Narimantas Evaldas Samalavicius • Darius Kazanavicius • Raimundas Lunevicius • \\ Tomas Poskus · Jonas Valantinas · Juozas Stanaitis · Aurelijus Grigaliunas • \\ Audrius Gradauskas • Donatas Venskutonis - Remigijus Samuolis • \\ Pranas Sniuolis • Mindaugas Gajauskas • Nerijus Kaselis • Raimundas Leipus • \\ Gintautas Radziunas
}

Published online: 29 June 2013

(C) Springer Science+Business Media New York 2013

Erratum to: Surg Endosc (2013) 27:1628-1635
DOI 10.1007/s00464-012-2642-4

The correct affiliation for Donatas Venskutonis is Clinic of General Surgery, Lithuanian University of Health Sciences, Kaunas Clinical Hospital, Josvainiu str. 2, 47144 Kaunas, Lithuania.

The online version of the original article can be found under doi:10.1007/s00464-012-2642-4.

N. E. Samalavicius $(\bowtie) \cdot D$. Kazanavicius

Center of Oncosurgery, Institute of Oncology, Clinic of Internal Diseases, Family Medicine and Oncology of Medical Faculty, Vilnius University, 1 Santariskiu str., LT-08660 Vilnius,

Lithuania

e-mail: narimantas.samalavicius@vuoi.lt

D. Kazanavicius

e-mail: dkazanavicius@gmail.com

R. Lunevicius

University Hospitals Aintree, Aintree University Hospital NHS

Foundation Trust, Lower Lane, Liverpool L9 7AL, UK

T. Poskus

3rd Department of Surgery, Vilnius University Hospital

Santariskiu Klinikos Center Branch, Zygimantu g. 3,

01102 Vilnius, Lithuania

J. Valantinas $\cdot$ J. Stanaitis

Clinic of Gastroenterology, Nephrourology and Surgery,

Medical Faculty of Vilnius University, Vilnius University

Hospital Santariskiu Klinikos, 2 Santariskiu str.,

08661 Vilnius, Lithuania
A. Grigaliunas

Department of Surgery, Vilnius University Republican Hospital,

29 Siltnamiu str., 04130 Vilnius, Lithuania

A. Gradauskas

Clinic of Surgery, Vilnius University Hospital,

57 Antakalnio str., Vilnius, Lithuania

D. Venskutonis

Clinic of General Surgery, Lithuanian University of Health

Sciences, Kaunas Clinical Hospital, Josvainiụ str. 2,

47144 Kaunas, Lithuania

\section{R. Samuolis}

Department of Surgery, Panevezys Republican Hospital,

25 Smelynes str., 35144 Panevezys, Lithuania

P. Sniuolis

Department of Surgery, Klaipeda University Hospital,

41 Liepojos str., 92288 Klaipeda, Lithuania 
M. Gajauskas

Department of Endoscopy, Kaunas 3rd Hospital,

13 Hipodromo str., Kaunas, Lithuania

\section{N. Kaselis}

Department of Surgery, Klaipeda District Hospital,

3 S. Neries str., 92231 Klaipeda, Lithuania

\section{R. Leipus}

Department of Surgery, Utena Hospital, 3 Aukstakalnio str.,

28151 Utena, Lithuania

G. Radziunas

Medical Diagnostic Center, 32 V. Grybo str.,

10318 Vilnius, Lithuania 